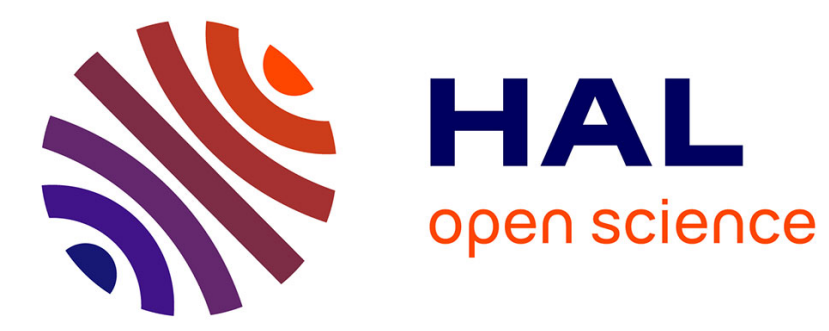

\title{
REVISITING THE CONNECTION BETWEEN THE NO-SHOW PARADOX AND MONOTONICITY *
}

\author{
Matías Núñez, Remzi Sanver
}

\section{To cite this version:}

Matías Núñez, Remzi Sanver. REVISITING THE CONNECTION BETWEEN THE NO-SHOW PARADOX AND MONOTONICITY *. 2016. hal-01276072v2

HAL Id: hal-01276072

https://hal.science/hal-01276072v2

Preprint submitted on 6 Jul 2016

HAL is a multi-disciplinary open access archive for the deposit and dissemination of scientific research documents, whether they are published or not. The documents may come from teaching and research institutions in France or abroad, or from public or private research centers.
L'archive ouverte pluridisciplinaire HAL, est destinée au dépôt et à la diffusion de documents scientifiques de niveau recherche, publiés ou non, émanant des établissements d'enseignement et de recherche français ou étrangers, des laboratoires publics ou privés. 


\title{
REVISITING THE CONNECTION BETWEEN THE NO-SHOW PARADOX AND MONOTONICITY*
}

\author{
MATÍAS NÚÑEZ ${ }^{a}$ AND M. REMZI SANVER ${ }^{a, b}$
}

\begin{abstract}
We investigate the relation between monotonicity and the no-show paradox in voting rules. Although the literature has established their logical independence, we show, by presenting logical dependency results, that the two conditions are closer than a general logical independency result would suggest. Our analysis is made both under variable and fixed-size electorates.

KeYwords. Monotonicity, No-Show Paradox, Participation, Reinforcement, Threshold Scoring Rules.

JEL Classification. D71.
\end{abstract}

\section{INTRODUCTION}

Among the countless contributions of Hervé Moulin to our enlightenment on the collective decision making problem, his research on the axiomatic analysis of social choice rules presents a distinguished chapter which inspired generations of scholars. We view this issue of Mathematical Social Sciences dedicated to him as a nice opportunity to revisit the connection between participation and monotonicity, two conditions of social choice theory which have been much elaborated by the fine work of Hervé Moulin.

Moulin [1988, 1991] defines participation as the vanishing of the no-show paradox introduced by Fishburn and Brams [1983]: a social choice rule exhibits the no-show paradox when the vote casted by an additional voter changes the outcome in a way which makes this new-comer worse off compared to the case he had not shown up. Thus, the paradox can be viewed as a way to manipulate social choice rules by abstaining to vote, such as Moulin [1991] who sees it as a particular case of manipulation by truncation of preferences as defined by Fishburn and Brams [1984].

a Université Paris-Dauphine, PSL Research University, CNRS, UMR [7243], LAMSADE, 75016 PARIS, FRANCE.

b Murat Sertel Center for Advanced Economic Studies, Istanbul Bilgi University, TURKEY.

* We acknowledge financial support from the ANR-14-CE24-0007-01 CoCoRICo-CoDec and from LAMSADE (Pôle 1). We also thank the Associate Editor and the referees for insightful comments that upgraded the quality of this work and the audiences at the Paris School of Economics and at the $13^{\text {th }}$ Meeting of the Society for Social Choice and Welfare, Lund, where this paper was presented for their suggestions. 
Such views, however, necessitate some caution on how the new-comer/abstainer is interpreted. Here, two approaches come to the fore: One is the fixed-electorate approach where the number of voters are fixed and the abstainers are those voters who express full indifference over the set of alternatives. So a "new-comer" is an individual who is an incumbent member of the society who moves away from his full indifference position. The other approach necessitates a variable-electorate, as the new-comer is a voter who earlier was not a member of the society, hence "abstaining" means his altogether departing from the society to which he used to be part of.

Rejecting to express a preference does not mean to leave the electorate. As a result, the two interpretations have different meanings. However, for social choice rules which are "regular", i.e., ignore voters who show up without expressing a preference, one could expect that the choice of the interpretation would not matter. Theorem 14 somehow justifies this expectation by establishing an equivalence between variable and fixed electorate social choice rules regarding the satisfaction of PART. ${ }^{1}$ On the other hand, the choice of the interpretation has implications on the relationship between the no-show paradox and monotonicity - a fact that we discuss in the sequel. However, we wish to note right away that the literature on the paradox has almost always adopted the variable-electorate approach, including and perhaps following the seminal paper of Moulin [1988]. ${ }^{2}$

We start, in Section 2, by considering the paradox under this standard variableelectorate interpretation and revisit its relation to a well-known monotonicity condition of social choice theory. Monotonicity, broadly speaking, requires that an "improvement" of the status of an alternative in the preferences of the electorate should result in a "raising" of the status of this alternative as the social outcome. It is clear that, different meanings can be attributed to "improvement" and "raising", each of which leading to a different definition of monotonicity. In fact, the literature exhibits a plethora of monotonicity conditions. As all of these can be connected to the (non)-manipulability of social choice rules, the logical relationship of participation to those monotonicity conditions stands out as an interesting question.

Among the various monotonicity conditions, perhaps the simplest and oldest known is the one we consider ${ }^{3}$ :

\footnotetext{
${ }^{1}$ While most of the well-known social choice rules are regular, there are notable exceptions such as those who use a quorum or those who allow voters to vote for "none of the above (NOTA)". A specific analysis of these rules, though out of the scope of this paper, can contribute to our understanding of the notion of abstention. We thank the associate editor who draw our attention to this.

${ }^{2}$ The paradox has also been considered in the framework of judgement aggregation (see Balinski and Laraki [2010]).

${ }^{3}$ For discussion on monotonicity conditions in social choice theory, one can see Fishburn [1982], Moulin [1983], Brams and Fishburn [2002] and Sanver and Zwicker [2009].
} 
MON: Raising an alternative $x$ in voters' preferences while leaving the rankings otherwise unchanged can never result in $x$ becoming the loser while $x$ was initially the winner.

Although normatively appealing and simple, MON is violated by various wellknown social choice rules, in particular by all point run-off systems (Smith [1973]). ${ }^{4}$ As participation (PART) is also violated by an interesting class of social choice rules, namely those which are Condorcet consistent (Moulin [1988]), the logical relationship between MON and PART turns out to be of further interest.

In fact, the general logical independence between MON and PART is already established. The question is addressed by Nurmi [1999] in p.62 who remarks that MON does not imply PART, as there exist Condorcet extensions, such as the Copeland rule, which satisfy MON but, by Moulin [1988], fail PART. Nurmi [1999] furthermore suggests the conjecture that PART implies MON which is falsified by Campbell and Kelly [2002] who give examples of social choice rules that satisfy PART but fail MON.

We present instances of logical dependencies between PART and MON. We show that in the particular case of two alternatives, PART implies MON. On the other hand, even with two alternatives, MON does not imply PART. Nevertheless, the failure of this implication merits some attention to the contextual difference regarding the definitions of the two conditions: while MON is a property that can be defined for fixed or variable electorate social choice rules, PART necessitates a variable electorate. As a result, PART requires a connection between how a social rule behaves in electorates of different sizes but MON does not. This renders the construction of a social choice rule which satisfies MON but fails PART very easy. In fact, the example we use in Proposition 1 to show that MON does not imply PART even with two alternatives exploits this ease.

A fairer question is whether MON implies PART under mild consistency requirements over the behavior of social choice rules in different electorates. One such condition is reinforcement, also known as consistency, which requires that alternatives which are separately chosen by both of two disjoint electorates must form the choice made by the union of these electorates (Smith [1973], Young [1974, 1975])). A much milder version of reinforcement is homogeneity which requires that an alternative which is chosen by some given electorate must also be chosen when this electorate is replicated. ${ }^{5}$ We show that in the two-alternative case, under the homogeneity assumption, MON implies PART.

\footnotetext{
${ }^{4}$ Other interesting violations of MON are established by Fishburn [1977], Richelson [1980],Fishburn and Brams [1983].

${ }^{5}$ Without omitting to note some borderline counter examples in Fishburn [1977], we can nevertheless say that almost all social choice rules considered in the literature are homogeneous.
} 
With three or more alternatives, Moulin [1988], while establishing the logical independence between PART and reinforcement, uses a threshold scoring rule to exemplify the satisfaction of reinforcement and the failure of PART. As all threshold scoring rules satisfy $\mathrm{MON}$, the example also shows that even when homogeneity is replaced by reinforcement, MON fails to imply PART.

Inspired by this example we devote further attention to threshold scoring rules ${ }^{6}$ and ask whether they always fail PART. The answer is almost affirmative: we show that, except one member, the class of threshold scoring rules fails PART.

We also consider a weaker version of participation (WPART) as the absence of a stronger version of the no-show paradox (Pérez [2001]) where a voter, by abstaining, can enforce his most preferred alternative as the social outcome. We show that MON, even when homogeneity is assumed, does not imply WPART. On the other hand, reinforcement, when combined with a weak unanimity condition, implies WPART.

Regarding the implication of monotonicity by PART, we show that PART implies a weaker version of monotonicity (WMON) which is nevertheless sufficiently strong to discriminate among social choice rules that fail MON: we observe that Campbell and Kelly [2002]'s examples that fail MON satisfy WMON while plurality with a runoff even fails WMON. In fact, we are able to extend this latter observation to the almost whole class of point runoff procedures which, except Borda, all fail WMON. Our Theorem 6 which states this failure not only strengthens the result of Smith (1973) on the failure of MON by point runoff procedures, but also paves the way to our Theorem 7 which announces that all point runoff procedures fail PART.

We close the section by giving a partial characterization of PART through a lower contour set intersection property which we call Condition $\lambda$. We also establish the relationship between Condition $\lambda$ and MON, which brings another perspective to our previous findings.

Section 3 carries our analysis to the fixed-electorate interpretation of PART. We start by establishing an equivalence between fixed and variable electorate interpretations regarding the satisfaction of PART. Based on this equivalence, we are able to note that the general logical independence between PART and MON prevails in the fixed electorate setting. On the other hand, regarding the logical relationship between PART and MON, our findings differ from those obtained under the variable-electorate interpretation. We show that with two alternatives, PART and MON are logically equivalent. Moreover, when three or more alternatives are available MON implies WPART and PART implies WMON.

Section 4 makes some closing remarks.

\footnotetext{
${ }^{6}$ One can see Saari [1990] for an analysis of these rules.
} 


\section{The Variable Population Case}

We consider a finite set of alternatives $A$ with $\# A \geq 2$. $\mathbf{N}$ denotes the set of natural numbers. For each $n \in \mathbf{N}$, we define $N=\{1, \ldots, n\} \subset \mathbf{N}$ as the $n$-voter electorate, where each $v \in N$ is a voter. $\Pi$ stands for the set of linear orders over $A . P_{v} \in \Pi$ is the preference of $v \in N$ over $A$, where for any distinct $x, y \in A, x P_{v} y$ indicates that voter $v$ prefers $x$ to $y{ }^{7}$ We write $P_{N}=\left\{P_{v}\right\}_{v \in N}$ for a preference profile over $A$. A social choice rule (SCR) is a mapping $F$ that returns, for each $n \in \mathbf{N}$ and each $P_{N} \in \Pi^{N}$, a single alternative $F\left(P_{N}\right) \in A$. So the SCRs we consider are variableelectorate in the sense of being defined for every number of voters and they satisfy the full domain condition in the sense that given any electorate, they are defined for every possible preference profile.

For any two electorates $N=\{1, \ldots, n\}$ and $M=\{1, \ldots, m\}$, we define the joint electorate $M \oplus N=\{1, \ldots, m+n\}$. Note that $\oplus$ is commutative. Now letting $m \leq n$, for any two profiles $R_{N}, Q_{M}$, we let $P_{N \oplus M}=\left(R_{N}, Q_{M}\right)$ stand for the profile of $M \oplus N$ where $P_{v}=R_{v} \forall v \in\{1, \ldots, n\}$ and $P_{n+v}=Q_{v} \forall v \in\{1, \ldots, m\}$. Note that when $m<n, P_{M \oplus N}$ is uniquely defined by setting the first $n$ voters as the voters of $N$ and the remaining $m$ voters as the voters of $M$. Abusing notation, when $M=\{v\}$, we write $\left(R_{N}, Q_{v}\right)$ to denote the profile obtained from $R_{N}$ by adding the preference $Q_{v}$ of voter $v$. Given any $n \in \mathbf{N}$ and $v \in N$, we let $N^{-v}=N \backslash\{v\}$.

Definition 1. A SCR $F$ satisfies participation (PART) iff $\forall N$ with $n \geq 2, \forall v \in N$, $\forall P_{N^{-v}}, \forall P_{v}$,

$$
F\left(P_{N^{-v}}, P_{v}\right) \neq F\left(P_{N^{-v}}\right) \Longrightarrow F\left(P_{N^{-v}}, P_{v}\right) P_{v} F\left(P_{N^{-v}}\right)
$$

Definition 2. Given any $N$, any $x$ and any $P_{N}, P_{N}^{\prime}$ such that $P_{v} \neq P_{v}^{\prime}$ for some $v \in N$ and $P_{w}=P_{w}^{\prime} \forall w \in N \backslash\{v\}$, we say that $P_{N}$ is an improvement for $x$ w.r.t. $P_{N}^{\prime}$ if

(1) $x P_{v}^{\prime} y \Longrightarrow x P_{v} y$ for every $y \in A \backslash\{x\}$,

(2) $y P_{v}^{\prime} z \Longleftrightarrow y P_{v} z$ for every $y, z \in A \backslash\{x\}$.

Definition 3. A SCR $F$ is monotonic (MON) iff given $x \in A, P_{N}, P_{N}^{\prime} \in \Pi^{N}$ such that $P_{N}$ is an improvement for $x$ w.r.t. $P_{N}^{\prime}$

$$
x=F\left(P_{N}^{\prime}\right) \Longrightarrow x=F\left(P_{N}\right){ }^{8}
$$

\footnotetext{
${ }^{7}$ Since $P_{v}$ is a linear order it is complete, asymmetric and transitive. So, by completeness, for any distinct $x, y \in A$, we have $x P_{v} y$ or $y P_{v} x$. Moreover, since $P_{v}$ is asymmetric, $\forall x, y \in A x P_{v} y \Longrightarrow y \neg P_{v} x$. Furthermore, if $x P_{v} y$ and $y P_{v} z$ then $x P_{v} z$ by transitivity.

${ }^{8}$ The definition of MON applies just to profiles that differ by a single voter's preference, since our focus is on its relation to PART which is defined with respect to the addition of a single voter. However, it should be noted that our definition is equivalent to the more common definition in the literature where MON applies also to profiles which possibly differ in a group of voters' preferences.
} 
2.1. The case of two alternatives. The logical independence between MON and PART vanishes when there are only two alternatives. In fact, in this case PART implies MON, as stated by the following theorem.

Theorem 1. Let $\# A:=2$. If a SCR F satisfies PART, then it satisfies MON.

Proof. Let $A:=\{x, y\}$. Take some $F$ which satisfies PART but fails MON. So $\exists N, v$, $P_{N^{-v}}, P_{v}, P_{v}^{\prime}$ with $x P_{v} y, y P_{v}^{\prime} x$ while $F\left(P_{N^{-v}}, P_{v}\right)=y$ and $F\left(P_{N^{-v}}, P_{v}^{\prime}\right)=x$. However, by PART, $F\left(P_{N^{-v}}, P_{v}\right)=y$ implies $F\left(P_{N^{-v}}\right)=y$ and $F\left(P_{N^{-v}}, P_{v}^{\prime}\right)=x$ implies $F\left(P_{N^{-v}}\right)=$ $x$, giving a contradiction.

Q.E.D.

The reverse implication holds, when a mild homogeneity condition is assumed. For any positive integer $m$, and any profile $P_{N}$, we write $m P_{N}$ for any of the profiles obtained from $P_{N}$ by replacing each single voter $v$ of $P_{N}$ with $m$ voters having the same preference as $v .^{9}$

Definition 4. A SCR F satisfies homogeneity (HOM) if $\forall N, \forall P_{N}, \forall m \in \mathbf{N}, \forall m P_{N}$, $F\left(m P_{N}\right)=F\left(P_{N}\right)$.

As the following theorem shows, any homogeneous SCR which satisfies MON satisfies PART as well.

Theorem 2. Let \#A $:=2$. If a SCR F satisfies HOM and MON, then it satisfies PART.

Proof. Let $A:=\{x, y\}$. Assume that $F$ satisfies HOM and MON but fails PART. Since $F$ fails PART, there is some $N$ with $\# N:=n \geq 2$, some profile $P_{N}$ and some voter $v \in N$ with $x P_{v} y$ while $F\left(P_{N^{-v}}\right)=x$ and $F\left(P_{N}\right)=y$. Let $\#\left\{v \in N \mid x P_{v} y\right\}:=k$ and $\#\left\{v \in N \mid y P_{v} x\right\}:=n-k$. Consider now the profiles $n P_{N^{-v}}$ and $(n-1) P_{N}$. Due to HOM, it follows that $F\left(n P_{N^{-v}}\right)=x$ and $F\left((n-1) P_{N}\right)=y$. Moreover, both profiles have the same number $n(n-1)$ of voters. Yet, they differ on the number of voters who prefer $x$ to $y$ : there are $n k-n$ voters who prefer $x$ to $y$ in $n P_{N^{-v}}$ and $n k-k$ in $(n-1) P_{N}$. Hence, there is some profile $(n-1) P_{N}$ which is an improvement for $x$ w.r.t. some profile $n P_{N^{-v}}$. Since $F\left(n P_{N^{-v}}\right)=x$, applying MON it follows that $F\left((n-1) P_{N}\right)=x$, giving a contradiction.

Q.E.D.

However, it should be noted that MON does not imply PART without HOM, as the following proposition shows.

Proposition 1. Let \#A :=2. There exists some $S C R F$ that satisfies $M O N$ and fails PART.

\footnotetext{
${ }^{9_{\text {Note }} \text { that } m} P_{N}$ is equivalent to $P_{N \oplus N \oplus \ldots \oplus N}$. As here, $\oplus$ is applied to sets of equal size, $m P_{N}$ is not uniquely defined.
} 
Proof. Let $A:=\{x, y\}$. We construct some $F$ that satisfies MON but fails PART (and HOM, by Theorem 2). When $\# N$ is even, let $F\left(P_{N}\right)=x$ iff $\#\left\{v \in N \mid x P_{v} y\right\} \geq$ $\#\left\{v \in N \mid y P_{v} x\right\}$. When $\# N$ is odd, let $F\left(P_{N}\right)=x$ iff $\#\left\{v \in N \mid x P_{v} y\right\}=\# N$. In other words, when $\# N$ is even, $F$ is the majority rule biased towards $x$ in the event of tie and when $\# N$ is odd $F$ is the unanimity rule biased towards $y$ in the absence of a unanimously agreed alternative. It is clear that $F$ satisfies MON. Let $P_{N}$ be a profile with two out of three voters who prefer $x$ to $y$ and one voter who prefers $y$ to $x$. Thus, there is some $P_{v} \in \Pi$ with $x P_{v} y$. It follows that $F\left(P_{N^{-v}}\right)=x$ and $F\left(P_{N}\right)=y$. Since $F\left(P_{N^{-v}}\right) P_{v} F\left(P_{N}\right), F$ violates PART as desired. To see that $F$ fails HOM, note that $F\left(2\left(P_{N}\right)\right)=x$ whereas $F\left(P_{N}\right)=y$.

Q.E.D.

\subsection{The case of three or more alternatives.}

2.2.1. On the implication of PART by MON. We start by showing that PART is not implied by MON even under the following reinforcement condition.

Definition 5. A SCR F satisfies reinforcement (REIN) if for any pair of electorates $M$ and $N$, for any $P_{M}, P_{N}$ and for any $x \in A$,

$$
F\left(P_{M}\right)=F\left(P_{N}\right)=x \Longrightarrow F\left(P_{M \oplus N}\right)=x .
$$

Assuming anonymity and neutrality, the conjunction of REIN with a few mild conditions characterizes scoring rules (Smith [1973], Young [1974, 1975], Myerson [1995]). In fact, one can see REIN as a core condition for being a scoring rule. On the other hand, Saari [1990] presents a weaker version of REIN and shows that threshold scoring rules represent a class of SCRs that fail REIN but satisfy its weak version. For single-valued SCRs, the strong and the weak versions of the condition coincide. Since scoring rules satisfy PART, we now show our claim through a threshold scoring rule.

For any preference $P_{v}$ and any alternative $x$, the rank of $x$ in $P_{v}$ equals $r\left(x ; P_{v}\right)=$ $1+\#\left\{y \in A \mid y P_{v} x\right\}$. A score vector $s=\left(s_{1}, \ldots, s_{\# A}\right)$ is an $\# A$-dimensional vector with $s_{1} \geq s_{2} \geq \ldots \geq s_{\# A}$ and $s_{1}>s_{\# A}$. Under a score vector $s$, the score of alternative $x$ at the preference profile $P_{N}$ equals $S\left(x ; P_{N} ; s\right)=\sum_{v \in N} s_{r\left(x ; P_{v}\right)}$. For any electorate $N$, any set $A$ of alternatives and any score vector $s$, we define a threshold $t(N, A, s):=$ $\frac{\# N}{\# A} \sum_{i=1}^{\# A} s_{i}$.

Definition 6. Every score vector $s$ induces a threshold scoring rule $F$ which is defined for every $N$ and every $P_{N}$ as $F\left(P_{N}\right)=\left\{x \in A \mid S\left(x ; P_{N} ; s\right) \geq t(N, A, s)\right\}$. When $F\left(P_{N}\right)$ is multi-valued, ties are broken according to an exogenous (alphabetical) linear order. 
Threshold scoring rules are well-defined since, by the choice of $t(N, A, s), F\left(P_{N}\right)$ is not empty for every $P_{N}$. The next lemma proves this point formally.

Lemma 1. If $F$ is a threshold scoring rule, $F\left(P_{N}\right)$ is not empty for every $P_{N}$.

Proof. Assume by contradiction that there is some profile $P_{N}$ for which $F\left(P_{N}\right)$ is empty. Thus, $S\left(x ; P_{N}, s\right)<t(N, A, s)$ for any $x \in A$. It follows that $\sum_{x \in A} S\left(x ; P_{N}, s\right)<$ $\sum_{x \in A} t(N, A, s)$. However, the left part of the inequality equals $\# N \sum_{i=1}^{\# A} s_{i}$ whereas the right part equals \#At$(N, A, s)$ which contradicts the definition of $t(N, A, s)$. Q.E.D.

Proposition 2. Let \#A $\geq 3$. There exists some SCR F that satisfies MON and REIN and fails PART.

Proof. Let $A:=\{x, y, z\}$ and consider $F$ to be the threshold scoring rule with $s=$ $(1,1,0)$. Hence, $t(N, A, s)=\frac{2}{3} n . F$ satisfies MON and REIN. To see that $F$ fails PART, let $N=\{1, \ldots, 6\}$ and take the preference profile $P_{N}$ with $a P_{v} b P_{v} c$ for $v=1,2$, $c P_{v} b P_{v} a$ for $v=3,4$ and $a P_{v} c P_{v} b$ for $v=5$, and $b P_{v} a P_{v} c$ for $v=6$. It follows that for $v=6, F\left(P_{N^{-v}}\right)=b$ and $F\left(P_{N}\right)=a$ while $F\left(P_{N^{-v}}\right) P_{v} F\left(P_{N}\right)$, proving that PART fails. ${ }^{10}$

Q.E.D.

We now ask whether all threshold scoring rules fail PART. The answer is almost affirmative as the theorem below shows.

Theorem 3. Let \#A $\geq 3$. Let $F$ be a threshold scoring rule induced by a score vector s. $F$ satisfies PART if and only if

$$
s=\left(s_{1}, \frac{s_{1}+s_{\# A}}{2}, \frac{s_{1}+s_{\# A}}{2}, \ldots, \frac{s_{1}+s_{\# A}}{2}, s_{\# A}\right) .
$$

Proof. Take some threshold scoring rule $F$ with score vector $s$. Suppose PART fails so $F\left(P_{N^{-v}}\right)=x$ and $F\left(P_{N^{-v}}, P_{v}\right)=y$ with $x P_{v} y$ for some $N, v, P_{N^{-v}}$ and $P_{v}$. This can occur under one of the following two exhaustive cases.

Case 1: $S\left(x, P_{N^{-v}}, s\right) \geq t\left(N^{-v}, A, s\right)$ and $S\left(x, P_{N}, s\right)<t(N, A, s)$.

Case 2: $\exists y \neq x$ with $S\left(y, P_{N^{-v}}, s\right)<t\left(N^{-v}, A, s\right)$ and $S\left(y, P_{N}, s\right) \geq t(N, A, s)$.

Note that $t(N, A, s)-t\left(N^{-v}, A, s\right)=\frac{\sum_{i=1}^{\# A} s_{i}}{\# A}$.

A necessary and sufficient condition to avoid case 1 is that the lowest additional score that $x$ receives with the arrival of $v$ is at least $\frac{\sum_{i=1}^{\# A} s_{i}}{\# A}$. As $x P_{v} y$, hence $r\left(x ; P_{v}\right) \geq$ $\# A-1$, this is ensured by setting $s_{\# A-1} \geq \frac{\sum_{i=1}^{\# A} s_{i}}{\# A}$.

A necessary and sufficient condition to avoid case 2 , it is that the highest additional score that $y$ gets with the arrival of $v$ does not exceed $\frac{\sum_{i=1}^{\# A} s_{i}}{\# A}$. As $x P_{v} y$, hence $r\left(y ; P_{v}\right) \leq 2$, this is ensured by setting $s_{2} \leq \frac{\sum_{i=1}^{\# A} s_{i}}{\# A}$.

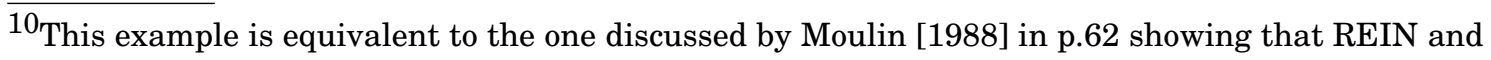
PART are logically independent.
} 
The two inequalities combined with $s_{2} \leq s_{3} \leq \ldots s_{\# A-1}$ imply $s_{j}=\frac{s_{1}+s_{\# A}}{2}$ for all $j=2, \ldots, \# A-1$.

Q.E.D.

Having shown that MON does not imply PART even under REIN, we now adopt a weaker version of participation, introduced by Pérez [2001], as the absence of a stronger version of the no-show paradox where a voter, by abstaining, can enforce his most preferred alternative as the social outcome.

Definition 7. A SCR F satisfies weak participation (WPART) iff $\forall N, \forall v \in N, \forall P_{N^{-v}}$, $\forall P_{v}$,

$$
F\left(P_{N^{-v}}, P_{v}\right) \neq F\left(P_{N^{-v}}\right) \Longrightarrow \exists x \in A \text { s.t. } x P_{v} F\left(P_{N^{-v}}\right) .
$$

Note that WPART is equivalent to PART with just two alternatives whereas it is weaker with more than two alternatives: PART requires that when adding the vote of $v$, voter $v$ prefers $F\left(P_{N^{-v}}, P_{v}\right)$ to $F\left(P_{N^{-v}}\right)$, whereas WPART just requires the existence of some alternative that $v$ prefers to $F\left(P_{N^{-v}}\right)$.

We now show that MON does not imply WPART either, even when combined with REIN.

Proposition 3. Let \#A $\geq 3$. There exists some SCR $F$ that satisfies REIN and MON but fails WPART.

Proof. Let $A:=\{x, y, z\}$ and consider the threshold scoring rule with $s=(1,1,0)$. This rule satisfies REIN and MON. In order to see why it fails WPART, consider the example used in the proof of Proposition 2. Since $b=F\left(P_{N^{-v}}\right) P_{v} F\left(P_{N^{-v}}, P_{v}\right)=$ $a$ with $b P_{v} a P_{v} c$, this shows that WPART fails.

Q.E.D.

Proposition 3 shows that MON and REIN do not imply WPART. However, when combined with the following weak unanimity condition imposed over singleton electorates, REIN implies WPART by its own.

Definition 8. A SCR $F$ is weakly unanimous iff $\forall v \in N, \forall P_{v}$,

$$
x P_{v} y \forall y \neq x \Rightarrow F\left(P_{v}\right)=x .
$$

Theorem 4. Let \#A $\geq 3$. If a weakly unanimous SCR F satisfies REIN, then it satisfies WPART.

Proof. Take some weakly unanimous $F$ that satisfies REIN. If it fails WPART, there exist $P_{N^{-v}}, P_{v}$ such that $F\left(P_{N^{-v}}, P_{v}\right)=x \neq F\left(P_{N^{-v}}\right)=z$ with $z P_{v} y \forall y \in A \backslash\{z\}$. As $z$ is first ranked in $P_{v}$, by weak unanimity, $F\left(P_{v}\right)=z$. By REIN, it follows that $F\left(P_{N^{-v}}, P_{v}\right)=z$, which leads to a contradiction.

Q.E.D.

It is worth noting that WPART is also studied by Richelson [1980] under the name "voter adaptability" and Theorem 4 is a precise expression of his statement in 
p.464 that "voter adaptability is a much weakened version of Young's consistency", as the validity of this claim requires weak unanimity. In fact, the threshold scoring rule in our Proposition 3 is not weakly unanimous; satisfies REIN (i.e., Young's consistency) and fails WPART.

2.2.2. On the implication of MON by PART. We ask whether MON has reasonable weakenings implied by PART. We first strengthen the definition of an improvement, by asking that the lifted alternative must be raised from the bottom of the ranking.

Definition 9. Given any $N$, any $x$ and any $P_{N}, P_{N}^{\prime}$ such that $P_{v} \neq P_{v}^{\prime}$ for some $v \in N$ and $P_{w}=P_{w}^{\prime} \forall w \in N \backslash\{v\}$, we say that $P_{N}$ is a strong improvement for $x$ w.r.t. $P_{N}^{\prime}$ if

(1) $y P_{v}^{\prime} x$ for every $y \in A \backslash\{x\}$,

(2) $y P_{v}^{\prime} z \Longleftrightarrow y P_{v} z$ for every $y, z \in A \backslash\{x\}$.

The following is a weakening of MON because the definition of improvement is strengthened but also because it allows alternatives above the lifted alternative to be chosen. As in the case of WPART and PART, it turns out that WMON and MON are equivalent with just two alternatives.

Definition 10. A SCR $F$ is weakly monotonic (WMON) iff given $x \in A, P_{N}, P_{N}^{\prime} \in \Pi^{N}$ such that $P_{N}$ is a strong improvement for $x$ w.r.t. $P_{N}^{\prime}$ :

$$
x=F\left(P_{N}^{\prime}\right) \text { and } F\left(P_{N}\right) \neq F\left(P_{N}^{\prime}\right) \Longrightarrow F\left(P_{N}\right) P_{v} x .
$$

Theorem 5. Let $\# A \geq 3$. If a SCR F satisfies PART, then it satisfies WMON.

Proof. Take some $F$ that satisfies PART but violates WMON. Since WMON fails, there exist some $N, v \in N, P_{N}, P_{N}^{\prime}$ with $P_{v}^{\prime} \neq P_{v}$ where $\left(P_{N^{-v}}, P_{v}\right)$ is a strong improvement for $x$ with respect to $\left(P_{N^{-v}}, P_{v}^{\prime}\right)$, while $F\left(P_{N^{-v}}, P_{v}^{\prime}\right)=x$ and $F\left(P_{N^{-v}}, P_{v}\right)=$ $y$ with $x P_{v} y$. Due to PART, $F\left(P_{N^{-v}}, P_{v}^{\prime}\right)=x$ implies $F\left(P_{N^{-v}}\right)=x$, as otherwise $F\left(P_{N^{-v}}\right) P_{v}^{\prime} x$ would hold, violating PART. Since $F\left(P_{N^{-v}}\right)=x$, then by PART again, we have $F\left(P_{N^{-v}}, P_{v}\right) \neq y$, giving a contradiction.

Q.E.D.

It should be noted that WMON is not too weak: it is able to discriminate among the SCRs that fail MON. For instance, one can check that the examples described in Campbell and Kelly [2002] which fail MON do satisfy WMON, which is the case by Theorem 5, as they all satisfy PART. On the other hand, plurality with a runoff, well known to fail MON, fails WMON as well, as we illustrate through the example below.

Example 1: Let $A:=\{x, y, z\}$ and consider two profiles $P_{N}^{\prime}, P_{N}$ with eight voters such that $P_{N}$ is a strong improvement for $z$ w.r.t. to $P_{N}^{\prime}$ : 


$$
\begin{aligned}
& P_{N}: \#\left\{v \in N \mid x P_{v} y P_{v} z\right\}=2, \#\left\{v \in N \mid y P_{v} z P_{v} x\right\}=2, \#\left\{v \in N \mid z P_{v} x P_{v} y\right\}=4, \\
& P_{N}^{\prime} \quad: \#\left\{v \in N \mid x P_{v}^{\prime} y P_{v}^{\prime} z\right\}=3, \#\left\{v \in N \mid y P_{v}^{\prime} z P_{v}^{\prime} x\right\}=2, \#\left\{v \in N \mid z P_{v}^{\prime} x P_{v}^{\prime} y\right\}=3 .
\end{aligned}
$$

Under plurality with a runoff where ties are broken in favor of $y$, at $P_{N}^{\prime}, x$ and $z$ which are both first ranked by three voters go for a runoff and, since there is a majority of voters who prefer $z$ to $x$, we have $F\left(P_{N}^{\prime}\right)=z$. At $P_{N}, z$ is first ranked by four voters whereas both $x$ and $y$ are ranked first by two voters each. As ties are broken in favor of $y, y$ and $z$ go for a runoff where $F\left(P_{N}\right)=y$. However, this violates WMON since $P_{N}$ is a strong improvement for $z$ w.r.t. to $P_{N}^{\prime}$ and $F\left(P_{N}^{\prime}\right)=z$.

In fact, the observation made by the example above reflects a more general fact: all point runoff procedures but one fail WMON. To see this, we first remark that when there are precisely three alternatives, every point runoff procedure eliminates the alternative with the lowest score according to some score vector $(1, \lambda, 0)$ with $0 \leq \lambda \leq 1$ (we assume that ties are broken alphabetically).

Theorem 6. Let \#A $\geq 3$. Every point runoff procedure fails WMON, unless $\lambda=\frac{1}{2}$.

Proof. Let $A:=\{x, y, z\}$ and consider some profile $P_{N}$ as follows, for some positive integers $n_{1}, n_{2}$ :

- three groups of $n_{1}$ voters, each group having respective preferences $x P_{v} y P_{v} z$, $y P_{v} z P_{v} x$ and $z P_{v} x P_{v} y$,

- three groups of $n_{2}$ voters, each group having respective preferences $x P_{v} z P_{v} y$, $z P_{v} y P_{v} x$ and $y P_{v} x P_{v} z$ and

- four voters, each with respective preference: $x P_{v} y P_{v} z, x P_{v} y P_{v} z, y P_{v} z P_{v} x$ and $z P_{v} x P_{v} y$.

There are, hence, $3 n_{1}+3 n_{2}+4$ voters in the profile $P_{N}$. It follows that there are $n_{1}-n_{2}+2$ more voters who prefer $x$ to $y$ than $y$ to $x$ and that there are $n_{1}-n_{2}$ more voters who prefer $z$ to $x$ than $x$ to $z$. We let $n_{1}>n_{2}$.

For any runoff procedure with vector $(1, \lambda, 0)$, the score that each alternative receives from the first six groups is equal to $\eta=\left(n_{1}+n_{2}\right)(1+\lambda)$. It follows that the score for the alternatives at $P_{N}$ equals $s(x)=\eta+2+\lambda, s(y)=\eta+1+2 \lambda$ and $s(z)=\eta+1+\lambda$ so that $s(x)>s(y) \geq s(z)$ as long as $\lambda<\frac{1}{2}$ and $s(x) \geq s(y)>s(z)$ when $\lambda>\frac{1}{2}$. Breaking ties alphabetically, $x, y$ go for a runoff and $x$ is the winner under any runoff procedure since $x$ is majority preferred to $y$ by the choice of $n_{1}, n_{2}$.

We now show that WMON fails as long as $\lambda \neq \frac{1}{2}$. We first analyze the case in which $\lambda<\frac{1}{2}$. 
Consider the profile $P_{N}^{\prime}$ such that the only difference with $P_{N}$ is that $k$ of the $n_{1}$ voters with preference $y P_{v} z P_{v} x$, raise $x$ to the top and switch to $x P_{v}^{\prime} y P_{v}^{\prime} z . P_{N}^{\prime}$ is a strong improvement for $x$ w.r.t. $P_{N}$. Letting $s^{\prime}(\cdot)$ be the scores of the alternatives under $P_{N}^{\prime}$ we have $\Delta(x)=s^{\prime}(x)-s(x)=k, \Delta(y)=s^{\prime}(y)-s(y)=k(1-\lambda)$ and $\Delta(z)=$ $s^{\prime}(z)-s(z)=k \lambda$. Note that when $\Delta(y)-\Delta(z)>\lambda$, hence $k(1-2 \lambda)>\lambda \Longleftrightarrow k>\frac{\lambda}{1-2 \lambda}, x$ and $z$ go for a runoff. It is possible to pick such a $k$ and $n_{1}-n_{2}$ arbitrarily large, so that $z$ is majority winner against $x$ at $P_{N}^{\prime}$ as well, showing that any runoff procedure with $\lambda<\frac{1}{2}$ fails WMON.

The case for $\lambda>\frac{1}{2}$ is similar to the previous one: it suffices to construct a profile $P_{N}^{\prime}$ such that the only difference with $P_{N}$ is that $k$ of the $n_{2}$ voters with preference $z P_{v} y P_{v} x$ in $P_{N}$ switch to $x P_{v}^{\prime} z P_{v}^{\prime} y$ in $P_{N}^{\prime}$. The above logic applies showing that any runoff procedure with $\lambda>\frac{1}{2}$ fails WMON as well.

The proof of Theorem 6 suggests that runoff procedures under Borda scores satisfy WMON, which we present as a conjecture. Nevertheless, we use Theorem 6 to show that no point runoff procedure satisfies PART.

Theorem 7. Let \#A $\geq 3$. Every point runoff procedure fails PART.

Proof. Note that, when $\# A=3$, any runoff procedure with $\lambda \neq \frac{1}{2}$ fails WMON (Theorem 6) and hence, due to Theorem 5, fails PART. Hence, the only runoff rule that might satisfy PART is the one with $\lambda=\frac{1}{2}$. To see that this rule also fails PART, take the profile $P_{N}$ as defined in the proof of Theorem 6 , where, letting $\lambda=\frac{1}{2}$, we have $s(x)=\eta+2.5, s(y)=\eta+2$ and $s(z)=\eta+1.5$ so that $x$ wins. Consider the profile $\left(P_{N}, P_{v}\right)$ where $x P_{v} z P_{v} y$. Now, the scores are $s^{\prime}(x)=\eta+3.5, s^{\prime}(y)=\eta+2$ and $s^{\prime}(z)=\eta+2$. If the tie between $y$ and $z$ is broken in favor of $z, x$ and $z$ go for a runoff, which leads to the victory of $z$, showing the failure of PART.

2.3. A partial characterization of PART. While we mainly focus on the connection between PART and MON, this section gives a partial characterization of PART through a lower contour set intersection property, which we call Condition $\lambda .^{11}$

For any $P_{v}$ and any alternative $x$, let $L\left(x ; P_{v}\right)=\left\{y \in A \mid x P_{v} y\right\}$ denote the set of alternatives such that $x$ is at least as good as any of them under $P_{v}$ (the lower contour set of $x$ under $P_{v}$ ).

Definition 11. A SCR F satisfies Condition $\lambda$ if for any $P_{N^{-v}}$ the following holds:

$$
\cap_{P_{v}} L\left(F\left(P_{N^{-v}} ; P_{v}\right), P_{v}\right) \neq \varnothing .
$$

Building on this condition, we now provide two sets of results: the first one deals with the relation of Condition $\lambda$ with PART whereas the second one focuses on its relation with MON.

\footnotetext{
${ }^{11}$ We thank the associate editor for suggesting this nice extension.
} 
To start with, we prove that PART and Condition $\lambda$ are almost equivalent.

Theorem 8. If a SCR F satisfies PART, then F satisfies Condition $\lambda$.

Proof. Take some $F$ that satisfies PART. Therefore, $\forall v \in N, \forall P_{N^{-v}}, \forall P_{v}, F\left(P_{N^{-v}}, P_{v}\right) \neq$ $F\left(P_{N^{-v}}\right) \Longrightarrow F\left(P_{N^{-v}}, P_{v}\right) P_{v} F\left(P_{N^{-v}}\right)$. It follows that $F\left(P_{N^{-v}}\right) \in L\left(F\left(P_{N^{-v}}, P_{v}\right) ; P_{v}\right)$ for every profile $P_{N^{-v}}$, thus Condition $\lambda$ holds.

It is hence clear that every scoring rule satisfies Condition $\lambda$ since any such rule satisfies PART. Yet, the same property does not apply to Condorcet extensions: as we now show, no such rule satisfies Condition $\lambda$.

Now, in order to state our claim, we introduce half-way monotonicity as defined by Sanver and Zwicker [2009]. For any linear order $P_{v}$ over $A$, we let $\operatorname{rev}\left(P_{v}\right)$ be the linear order obtained by reversing $P_{v}$ so that $x P_{v} y$ iff $y \operatorname{rev}\left(P_{v}\right) x$ for any pair $x, y$ of alternatives.

Definition 12. A SCR $F$ is half-way monotonic (HMON), if for any $N$, any $v \in N$, any $P_{v}, P_{N^{-v}}$ and any $x, y \in A$ :

$$
x=F\left(P_{N^{-v}}, P_{v}\right) \text { and } y=F\left(P_{N^{-v}}, \operatorname{rev}\left(P_{v}\right)\right) \Longrightarrow x P_{v} y .
$$

HMON can be interpreted as follows: a rule that violates HMON can be manipulated by some voter who completely misrepresents his preference, in the sense of announcing a preference that reverses every possible pairwise comparison among alternatives.

Lemma 2. If a SCR F satisfies Condition $\lambda$, then $F$ satisfies HMON.

Proof. Take some $F$ that fails $H M O N$. Therefore, there exists some $P_{N^{-v}}$ and some pair $x, y \in A$ with $x=F\left(P_{N^{-v}}, P_{v}\right)$ and $y=F\left(P_{N^{-v}}, \operatorname{rev}\left(P_{v}\right)\right)$ with $y P_{v} x$. However, $L\left(x ; P_{v}\right) \cap L\left(y ; \operatorname{rev}\left(P_{v}\right)\right)=\varnothing$ since $\operatorname{rev}\left(P_{v}\right)$ is the reversal of $P_{v}$ and $y P_{v} x$. Hence, $F$ fails Condition $\lambda$, concluding the proof.

Theorem 9. No Condorcet extension satisfies Condition $\lambda$.

Proof. Since no Condorcet extension satisfies HMON with four or more alternatives and sufficiently many voters (see Corollary 5.3 in Sanver and Zwicker [2009]) and Condition $\lambda$ implies HMON, it follows that no Condorcet extension satisfies Condition $\lambda$.

As a corollary to Theorem 8 and Theorem 9, we obtain Moulin [1988]'s result that no Condorcet extension satisfies PART.

The converse of Theorem 8 does not hold as proved by the next result.

Proposition 4. Let \#A $\geq 3$. There exists some SCR $F$ that satisfies Condition $\lambda$ and fails WPART, hence PART. 
Proof. Fix a pair of alternatives $x, y$ and some voter $d$. Consider the following SCR $F$ : (1) if $d$ takes part in the election, then $F$ chooses $d$ 's most preferred alternative among $\{x, y\}$ whereas (2) when $d$ does not take part in the election, $F$ chooses the winner according to Plurality voting. One can check that $F$ fails PART. In order to see why $F$ satisfies Condition $\lambda$, take first any profile $P_{N}$ where $d$ takes part in the election. Since the outcome $F\left(P_{N}\right)$ is the most preferred one of $d$ over $\{x, y\}$, Condition $\lambda$ holds. Take now a profile $P_{N}$ without $d$ so that the outcome $F\left(P_{N}\right)$ is determined through Plurality rule. Since this rule satisfies PART, it also satisfies Condition $\lambda$ as proved by Theorem 8 . Therefore, $F$ satisfies Condition $\lambda$.

Now, in order to prove that the converse of Theorem 8 holds provided that some mild conditions are added, we introduce the reversal cancellation property. Reversal cancellation, as defined by Sanver and Zwicker [2009], is arguably quite mild: according to it, adding a linear order and its reversal should leave the outcome of the SCR unchanged.

Definition 13. A SCR F satisfies Reversal Cancellation $(R C)$ if for any $N$, any $v \in N$, any $P_{v}$ and any $P_{N} \in \Pi^{N}$ :

$$
F\left(P_{N}\right)=F\left(P_{N}, P_{v}, \operatorname{rev}\left(P_{v}\right)\right)
$$

The next proposition shows that, combined with HOM and RC, Condition $\lambda$ implies PART.

Theorem 10. Let \#A $\geq 3$. If a SCR F satisfies Condition $\lambda, H O M$ and $R C$, then $F$ satisfies PART.

Proof. Take some SCR $F$ that fails PART. Therefore, there exists some $P_{N^{-v}}$ and $P_{v}$ with $x=F\left(P_{N^{-v}}\right) P_{v} F\left(P_{N^{-v}}, P_{v}\right)=y$ for some $x, y$. If $F$ satisfies HOM and RC, then it follows that $F\left(P_{N^{-v}}\right)=F\left(2 P_{N^{-v}}\right)=F\left(2 P_{N^{-v}}, P_{v}, \operatorname{rev}\left(P_{v}\right)\right)$ and $F\left(P_{N^{-v}}, P_{v}\right)=$ $F\left(2 P_{N^{-v}}, P_{v}, P_{v}\right)$. It follows that $F\left(2 P_{N^{-v}}, P_{v}, \operatorname{rev}\left(P_{v}\right)\right)=x$ and $F\left(2 P_{N^{-v}}, P_{v}, P_{v}\right)=y$. However, $L\left(x ; \operatorname{rev}\left(P_{v}\right)\right) \cap L\left(y ; P_{v}\right)=\varnothing$ since $x P_{v} y$ by definition and $\operatorname{rev}\left(P_{v}\right)$ is the reversal linear order of $P_{v}$. Hence $F$ fails Condition $\lambda$, which concludes the proof.

To see why RC cannot be dropped with more than three alternatives, it suffices to consider the voting rule described by the proof of Proposition 4: this rule satisfies HOM and Condition $\lambda$ but fails both RC and PART. With two alternatives, RC is not anymore needed. Indeed, Condition $\lambda$ is equivalent to MON as will be shown by Theorem 12 below. Therefore, using Theorem 2, one can see that Condition $\lambda$ jointly with HOM implies PART in this case. 
Once we have shown the almost equivalence of PART and Condition $\lambda$, this final set of results studies the relationship between Condition $\lambda$ and MON. We first show that both conditions are logically independent with at least three alternatives.

Theorem 11. Let $\# A \geq 3$. MON and Condition $\lambda$ are logically independent.

Proof. In order to check that MON does not imply Condition $\lambda$, it suffices to see any Condorcet extension that satisfies MON, e.g. Copeland rule, must fail Condition $\lambda$, as proved by Theorem 9 .

Now, in order to see that Condition $\lambda$ does not imply MON, note that Theorem 8 shows that PART implies Condition $\lambda$. Hence, combining this observation with the examples provided by Campbell and Kelly [2002] that satisfy PART but fail MON, concludes the proof.

Even though the conditions are logically independent, we can prove that they are equivalent with just two alternatives and that Condition $\lambda$ generally implies WMON, the weaker notion of monotonicity.

Theorem 12. Let \#A $:=2$. A SCR $F$ satisfies Condition $\lambda$ if and only if $F$ satisfies $M O N$.

Proof. Let $A:=\{x, y\}$. Take some $F$ that satisfies Condition $\lambda$ but fails MON. So, $\exists N, v, P_{N^{-v}}, P_{v}, P_{v^{\prime}}$ with $x P_{v} y, y P_{v^{\prime}} x$ while $F\left(P_{N^{-v}}, P_{v}\right)=y$ and $F\left(P_{N^{-v}}, P_{v^{\prime}}\right)=x$. However, this implies that $L\left(y ; P_{v}\right)=\{y\}$ whereas $L\left(x ; P_{v^{\prime}}\right)=\{x\}$ so that Condition $\lambda$ fails, a contradiction.

Take now some $F$ that satisfies MON. So, $\exists N, v, P_{N^{-v}}, P_{v}, P_{v^{\prime}}$ with $L\left(F\left(P_{N^{-v}}, P_{v}\right) ; P_{v}\right) \cap$ $L\left(F\left(P_{N^{-v}}, P_{v^{\prime}}\right) ; P_{v^{\prime}}\right)=\varnothing$. Assume w.l.o.g. that $x P_{v} y$ and $y P v x$. Then if $F\left(P_{N^{-v}}, P_{v}\right)=$ $x, L\left(F\left(P_{N^{-v}}, P_{v}\right) ; P_{v}\right)=\{x, y\}$ so that Condition $\lambda$ holds. If $F\left(P_{N^{-v}}, P_{v}\right)=y$, then $L\left(F\left(P_{N^{-v}}, P_{v}\right) ; P_{v}\right)=\{y\}$. If, now $F\left(P_{N^{-v}}, P_{v^{\prime}}\right)=y$ then $L\left(F\left(P_{N^{-v}}, P_{v^{\prime}}\right) ; P_{v^{\prime}}\right)=\{x, y\}$ so that Condition $\lambda$ holds again. Therefore, it must be the case that $F\left(P_{N^{-v}}, P_{v^{\prime}}\right)=x$ to ensure that Condition $\lambda$ holds. But this contradicts MON since $x P_{v} y$ and $y P v x$, concluding the proof.

Combining Theorem 12 with Theorem 1, Theorem 2 and Proposition 1, one can see that with just two alternatives, PART implies Condition $\lambda$; Condition $\lambda$ does not imply PART and condition $\lambda$ together with HOM imply PART.

Theorem 13. If a SCR F satisfies Condition $\lambda$, then it satisfies WMON.

Proof. Take some SCR $F$ that fails WMON but satisfies Condition $\lambda$. Since $F$ fails WMON, there must exist $P_{N^{-v}}, P_{v}, P_{v}^{\prime}$ and $x, y$ such that $F\left(P_{N^{-v}}, P_{v}\right)=x$ and $F\left(P_{N^{-v}}, P_{v}^{\prime}\right)=y$ with $x$ ranked last at $P_{v}$ and $y$ ranked below $x$ at $P_{v}^{\prime}$. Hence $L\left(x, P_{v}\right)=\{x\}$ whereas $x \notin L\left(y, P_{v}^{\prime}\right)$ since $y$ ranked below $x$. But this implies that 
$F$ fails Condition $\lambda$ since the previous equalities imply that $L\left(F\left(P_{N^{-v}} ; P_{v}\right), P_{v}\right) \cap$ $L\left(F\left(P_{N^{-v}}, P_{v}^{\prime}\right) ; P_{v}^{\prime}\right)=\varnothing$.

\section{The Fixed Electorate Case}

We now consider the case where the electorate $N$ is of fixed size $n \geq 2$. A voter $v$ is now allowed to have as preference a linear order $P_{v} \in \Pi$ or to abstain, i.e. have full indifference over the whole set of alternatives. This indifference is denoted by the null preference $R_{0}$ where $x R_{0} y$ holds for any $x, y \in A$. We let $\bar{\Pi}:=\Pi \cup\left\{R_{0}\right\}$. The profile $\left(P_{N^{-v}}, R_{0}\right) \in \bar{\Pi}^{N}$ is the profile in which voter $v$ abstains and the rest of voters' preferences are as in $P_{N^{-v}}$.

Given any $n \in \mathbf{N}$, a fixed-size social choice rule (FSCR) is a mapping $F_{n}$ that returns, for $P_{N} \in \bar{\Pi}^{N}$, a single alternative $F_{n}\left(P_{N}\right) \in A$. Note that the full domain assumption prevails, i.e. given the fixed electorate $N, F_{n}$ is defined for every possible preference profile $P_{N}$.

We now define MON under the possibility of abstention in individual preferences.

Definition 14. Given any $x$ and any $P_{N}, P_{N}^{\prime}$ with $P_{v} \neq P_{v}^{\prime}$ for some $v \in N$ and $P_{w}=$ $P_{w}^{\prime} \forall w \in N \backslash\{v\}$, If $P_{v}^{\prime} \neq R_{0}$, then $P_{N}$ is an improvement for $x$ w.r.t. $P_{N}^{\prime}$ if

(1) $x P_{v}^{\prime} y \Longrightarrow x P_{v} y$ for every $y \in A \backslash\{x\}$,

(2) $y P_{v}^{\prime} z \Longleftrightarrow y P_{v} z$ for every $y, z \in A \backslash\{x\}$.

If $P_{v}^{\prime}=R_{0}$, then $P_{N}$ is an improvement for $x$ w.r.t. $P_{N}^{\prime}$ if

(1) $x P_{v} y$ for every $y \in A \backslash\{x\}$.

Definition 15. A FSCR $F_{n}$ is monotonic (MON) iff given $x \in A, P_{N}, P_{N}^{\prime} \in \bar{\Pi}^{N}$ such that $P_{N}$ is an improvement for $x$ w.r.t. $P_{N}^{\prime}$,

$$
x=F_{n}\left(P_{N}^{\prime}\right) \Longrightarrow x=F_{n}\left(P_{N}\right) .
$$

We now define PART in this framework.

Definition 16. A FSCR $F_{n}$ satisfies participation (PART) iff $\forall v \in N, \forall P_{N^{-v}} \in \bar{\Pi}^{N^{-v}}$, $\forall P_{v} \in \bar{\Pi}$,

$$
F_{n}\left(P_{N^{-v}}, P_{v}\right) \neq F_{n}\left(P_{N^{-v}}, R_{0}\right) \Longrightarrow F_{n}\left(P_{N^{-v}}, P_{v}\right) P_{v} F_{n}\left(P_{N^{-v}}, R_{0}\right) \text {. }
$$

We now establish an equivalence between the fixed and variable electorate interpretations regarding the satisfaction of PART. We start by giving two definitions.

Definition 17. A family of FSCRs $\left\{F_{n}\right\}_{n \in \mathbf{N}}$ is equivalent to a variable electorate $S C R$ $F$ if and only if for any $n \in \mathbf{N}$ and any $P_{N} \in \Pi^{N}, F_{n}\left(P_{N}\right)=F\left(P_{N}\right)$.

Definition 18. A family of FSCRs $\left\{F_{n}\right\}_{n \in \mathbf{N}}$ is regular if for any $n \in \mathbf{N}$, for any $P_{N} \in$ $\bar{\Pi}^{N}$ with $P_{i}=R_{0}$ for some $i \in\{1, \ldots, n\}, F_{n}\left(P_{N}\right)=F_{n}\left(P_{1}, P_{2}, \ldots, P_{i-1}, P_{i+1}, \ldots, P_{n}\right)$. 
The meaning of Definition 17 is clear. The idea behind regularity is to ignore voters who take part in the election without expressing a preference; this is satisfied by many well-known rules such as scoring rules and the Condorcet principle. Nevertheless, the quorum rules (see Houy [2009]), where some minimal level of participation is required, fail to satisfy regularity.

Observe that for each variable electorate SCR $F$, there exists some equivalent family $\left\{F_{n}\right\}_{n \in \mathbf{N}}$ of FSCRs which is not unique. Yet, uniqueness is obtained when regularity is imposed over $\left\{F_{n}\right\}_{n \in \mathbf{N}}$.

Theorem 14. Let $F$ be a variable electorate $S C R$ and $\left\{F_{n}\right\}_{n \in \mathbf{N}}$ the regular family of FSCRs which is equivalent to $F$. F satisfies PART if and only if $F_{n}$ satisfies PART for each $n \in \mathbf{N}$.

Proof. Take some SCR $F$ and its equivalent regular family of $F S C R s\left\{F_{n}\right\}_{n \in \mathbf{N}}$.

Assume that $F$ satisfies PART. Due to Definition 17, it follows that for any $n \geq 2$, $\forall v \in N, \forall P_{N^{-v}}, \forall P_{v}$,

$$
F\left(P_{N^{-v}}, P_{v}\right)=F_{n}\left(P_{N^{-v}}, P_{v}\right) \text { and } F\left(P_{N^{-v}}\right)=F_{n-1}\left(P_{N^{-v}}\right) .
$$

Moreover, Definition 18 implies that $F_{n-1}\left(P_{N^{-v}}\right)=F_{n}\left(P_{N^{-v}}, R_{0}\right)$. Since $F$ satisfies PART, it follows that $F\left(P_{N^{-v}}, P_{v}\right) \neq F\left(P_{N^{-v}}\right) \Longrightarrow F\left(P_{N^{-v}}, P_{v}\right) P_{v} F\left(P_{N^{-v}}\right)$. Therefore, the previous equalities imply that, as long as $F_{n}\left(P_{N^{-v}}, P_{v}\right) \neq F_{n}\left(P_{N^{-v}}, R_{0}\right)$,

$$
F_{n}\left(P_{N^{-v}}, P_{v}\right) P_{v} F_{n}\left(P_{N^{-v}}, R_{0}\right),
$$

which proves that $F_{n}$ satisfies PART for any $n \in \mathbf{N}$.

Assume now that each $F_{n}$ satisfies PART. Again Definitions 17 and 18 jointly imply that

$$
F\left(P_{N^{-v}}, P_{v}\right)=F_{n}\left(P_{N^{-v}}, P_{v}\right) \text { and } F\left(P_{N^{-v}}\right)=F_{n}\left(P_{N^{-v}}, R_{0}\right) .
$$

Since each $F_{n}$ satisfies PART, it follows that

$$
F_{n}\left(P_{N^{-v}}, P_{v}\right) \neq F_{n}\left(P_{N^{-v}}, R_{0}\right) \Longrightarrow F_{n}\left(P_{N^{-v}}, P_{v}\right) P_{v} F_{n}\left(P_{N^{-v}}, R_{0}\right) .
$$

However, combining the previous implication with the described equivalence between the SCR $F$ and each FSCR $F_{n}$, proves that $F$ satisfies PART, as desired.

Q.E.D.

By using Theorem 14 we can transfer results on the satisfaction of PART in the variable electorate setting to the fixed electorate one. More precisely, Moulin [1988] proves that with four or more alternatives and with at least 25 voters, no Condorcet rule satisfies PART. Recently, Brandt et al. [2016] proves that the minimal number of voters to obtain this incompatibility is exactly 12 using computational 
techniques ${ }^{12}$. As a consequence of these results, we can conclude that scoring rules satisfy PART in the fixed electorate setting as well or that the result of Moulin on the non-existence of Condorcet extensions that satisfy PART prevails when the electorate is fixed. To be more precise regarding the latter observation, there exists some $n \in \mathbf{N}$ where no FSCR $F_{n}$ is Condorcet Consistent and satisfies PART. This also shows that MON does not imply PART in this setting as well, as a monotonic Condorcet extension such as the Copeland rule illustrates. As a matter of fact, the general logical independence between PART and MON prevails in this setting as shown by the following adaptation of the Campbell and Kelly [2002] example to the fixed electorate setting.

Example: PART does not imply MON. Fix some $x \in A$. We define a FSCR $F_{n}$ such that, for any profile $P_{N}$, (1) if $P_{v}=R_{0}$ for every $v \in N$, then $F_{n}\left(P_{N}\right)=x$, (2) if $x$ is ranked last by every $P_{v} \neq R_{0}$, then $F_{n}\left(P_{N}\right)=x$ and (3) if there is some $P_{v} \neq R_{0}$ where some alternative $y$ different from $x$ is ranked last, then $F_{n}$ selects the most preferred alternative of the voter with the lowest index among those with a strict preference where $x$ is not ranked last. One can check that $F_{n}$ satisfies PART but fails MON.

As the following theorem states, for FSCRs, MON and PART are equivalent when there are two alternatives only.

Theorem 15. Let $\# A:=2$. A FSCR $F_{n}$ satisfies MON if and only if it satisfies PART.

Proof. Let $A:=\{x, y\}$. Take some $F_{n}$ that satisfies PART but fails MON. Since $F_{n}$ fails MON, w.l.o.g., one of the following two exhaustive cases holds.

Case 1: there exist some profile $P_{N^{-v}} \in \bar{\Pi}^{N^{-v}}$ and some pair $P_{v}^{\prime}, P_{v}$ with $y P_{v}^{\prime} x$ and $x P_{v} y$ with $F_{n}\left(P_{N^{-v}}, P_{v}^{\prime}\right)=x$ and $F_{n}\left(P_{N^{-v}}, P_{v}\right)=y$. However, due to PART, $F_{n}\left(P_{N^{-v}}, P_{v}^{\prime}\right)=$ $x$ implies $F_{n}\left(P_{N^{-v}}, R_{0}\right)=x$ which in turn implies $F_{n}\left(P_{N^{-v}}, P_{v}\right)=x$, giving a contradiction.

Case 2: there exist some profile $P_{N^{-v}} \in \bar{\Pi}^{N^{-v}}$ and some pair $P_{v}^{\prime}=R_{0}, P_{v}$ with $x P_{v} y$ with $F_{n}\left(P_{N^{-v}}, P_{v}^{\prime}\right)=x$ and $F_{n}\left(P_{N^{-v}}, P_{v}\right)=y$. However, as $F_{n}\left(P_{N^{-v}}, R_{0}\right)=x$, PART implies $F_{n}\left(P_{N^{-v}}, P_{v}\right)=x$, giving a contradiction. We leave the reader to check that MON implies PART.

Q.E.D.

We now define WPART in the fixed electorate framework.

Definition 19. A FSCR $F_{n}$ satisfies weak participation (WPART) iff $\forall v \in N, \forall P_{N^{-v}} \in$ $\bar{\Pi}^{n-1}, \forall P_{v} \in \bar{\Pi}$,

$$
F_{n}\left(P_{N^{-v}}, P_{v}\right) \neq F_{n}\left(P_{N^{-v}}, R_{0}\right) \Longrightarrow \exists x \in A \text { s.t. } x P_{v} F_{n}\left(P_{N^{-v}}, R_{0}\right) .
$$

\footnotetext{
$\overline{12}$ For a recent contribution on the No-Show paradox with social choice correspondences, see Brandl et al. [2015].
} 
Theorem 16. Let \#A $\geq 3$. If a FSCR $F_{n}$ satisfies MON, then it satisfies WPART.

Proof. Take some $F_{n}$ that fails WPART. Since $F_{n}$ fails WPART, there must some profile $P_{N^{-v}} \in \bar{\Pi}^{N^{-v}}$ and some pair $P_{v}^{\prime}, P_{v}=R_{0}$ with $x P_{v}^{\prime} z \forall z \in A \backslash\{x\}$ while $F_{n}\left(P_{N^{-v}}, P_{v}\right)=$ $x$ and $F_{n}\left(P_{N^{-v}}, P_{v}^{\prime}\right) \neq x$. As $\left(P_{N^{-v}}, P_{v}^{\prime}\right)$ is an improvement for $x$ w.r.t. $\left(P_{N^{-v}}, P_{v}\right)$, this violates MON and concludes the proof.

Q.E.D.

The following is the weakening of MON in the same spirit as the weakening introduced in Section 2.

Definition 20. Given any $x$ and any $P_{N}, P_{N}^{\prime}$ such that $P_{v} \neq P_{v}^{\prime}$ for some $v \in N$ and $P_{w}=P_{w}^{\prime} \forall w \in N \backslash\{v\}$, If $P_{v}^{\prime} \neq R_{0}$, then $P_{N}$ is a strong improvement for $x$ w.r.t. $P_{N}^{\prime}$ if

(1) $y P_{v}^{\prime} x$ for every $y \in A \backslash\{x\}$,

(2) $y P_{v}^{\prime} z \Longleftrightarrow y P_{v} z$ for every $y, z \in A \backslash\{x\}$.

If $P_{v}^{\prime}=R_{0}$, then $P_{N}$ is a strong improvement for $x$ w.r.t. $P_{N}^{\prime}$ if

(1) $x P_{v} y$ for every $y \in A \backslash\{x\}$.

Definition 21. A FSCR $F_{n}$ is weakly monotonic (WMON) iff given $x \in A, P_{N}, P_{N}^{\prime} \in$ $\bar{\Pi}^{N}$ such that $P_{N}$ is a strong improvement for $x$ w.r.t. $P_{N}^{\prime}$

$$
x=F_{n}\left(P_{N}^{\prime}\right) \text { and } F_{n}\left(P_{N}\right) \neq F_{n}\left(P_{N}^{\prime}\right) \Longrightarrow F_{n}\left(P_{N}\right) P_{v} x .
$$

Theorem 17. Let \#A $\geq 3$. If a FSCR $F_{n}$ satisfies PART, then it satisfies WMON.

Proof. Take some $F_{n}$ that satisfies PART but fails WMON. Since $F_{n}$ fails WMON, one of the following two exhaustive cases holds.

Case 1: there exist some profile $P_{N^{-v}} \in \bar{\Pi}^{N^{-v}}$ and some pair $P_{v}^{\prime}, P_{v}$ with $z P_{v}^{\prime} x \forall z \in$ $A \backslash\{x\}$ and $x P_{v} y$ with $F_{n}\left(P_{N^{-v}}, P_{v}^{\prime}\right)=x$ and $F_{n}\left(P_{N^{-v}}, P_{v}\right)=y$. However, due to PART, $F_{n}\left(P_{N^{-v}}, P_{v}^{\prime}\right)=x$ implies $F_{n}\left(P_{N^{-v}}, R_{0}\right)=x$ which in turn implies $F_{n}\left(P_{N^{-v}}, P_{v}\right) \neq y$, giving a contradiction.

Case 2: there exist some profile $P_{N^{-v}} \in \bar{\Pi}^{N^{-v}}$ and some pair $P_{v}^{\prime}=R_{0}, P_{v}$ with $x P_{v} z \forall z \neq x$ while $F_{n}\left(P_{N^{-v}}, P_{v}^{\prime}\right)=x$ and $F_{n}\left(P_{N^{-v}}, P_{v}\right)=y$. However, due to PART, $F_{n}\left(P_{N^{-v}}, R_{0}\right)=x$ implies $F_{n}\left(P_{N^{-v}}, P_{v}\right)=x$, giving a contradiction.

Thus, there is no $F_{n}$ satisfying PART but failing WMON, which concludes the proof.

Q.E.D.

\section{Concluding REMARKS}

Although the logical independence between the no-show paradox and the failure of monotonicity has already been observed, our findings suggest that this observation does not mean a major conceptual gap between the two conditions. In fact, 
under two different interpretations of "a new-comer to the society", we are able to present instances where participation and monotonicity get very close to each other - in some particular cases to the extent that the established general logical independence between them vanishes.

This closeness is rather expected to us because, as we discuss in the introduction, both conditions are related to the manipulability of SCRs. In fact, until their logical independence was established by Nurmi [1999] and Campbell and Kelly [2002], there was a prevailing intuition that the two conditions were somehow related, in particular that PART could imply MON (see, for example, Nurmi [1999], p.62). Our findings point to a wisdom in this intuition: Although Campbell and Kelly [2002] show that PART does not imply MON, as the unorthodoxy of their examples suggests, PART almost implies MON, more precisely implies its weaker version WMON. On the other hand, as our Proposition 3 suggests, the fact that MON does not imply PART is not a mere consequence of the fact that PART is a condition for SCRs defined over variable size societies while MON is not.

Our discussions on the relationship between PART and MON paved the way to general results on certain interesting classes of SCRs. In particular, we show that all threshold scoring rules but one fail PART; all point runoff procedures but one (namely Borda) fail WMON; and all point runoff procedures fail PART.

It is worth noting that although the fixed-electorate interpretation has no considerable effect on the class of social choice rules that satisfy PART, it results in MON and PART getting closer. This is also rather expected because, again as discussed in the introduction, under this interpretation the link between the no-show paradox and manipulability of SCRs is more direct. A point we wish to emphasize is the equivalence between PART and MON under the fixed-electorate interpretation, when there are two alternatives. In this framework, majority rules with quorums are known to fail MON and they are supposed to give room to manipulation by abstention (see Houy [2009]). Our Theorem 15 is a formal expression of this supposition.

Finally, we wish to remark that PART has been mostly considered in the literature for single-valued SCRs which led our analysis to be held in this framework. However, there are a relatively few considerations of PART for multi-valued SCRs, such as Jimeno et al. [2009], and how our analysis would carry to that framework remains as an open question.

\section{REFERENCES}

Michel L. Balinski and Rida Laraki. Majority judgment: measuring, ranking, and electing. MIT press, 2010. 
REVISITING THE CONNECTION BETWEEN THE NO-SHOW PARADOX AND MONOTONICITY 21

Steven J. Brams and Peter C. Fishburn. Voting procedures. Handbook of Social Choice and Welfare, 1:173-236, 2002.

F. Brandl, F. Brandt, C. Geist, and J. Hofbauer. Strategic abstention based on preference extensions: Positive results and computer-generated impossibilities. Proceeding IJCAI'15 Proceedings of the 24th International Conference on Artificial Intelligence, pages 18-24, 2015.

F. Brandt, C. Geist, and D. Peters. Optimal bounds for the no-show paradox. In Proceedings of the 15th International Conference on Autonomous Agents and Multiagent Systems (AAMAS), 2016.

Donald E. Campbell and Jerry S. Kelly. Non-monotonicity does not imply the noshow paradox. Social Choice and Welfare, 19(3):513-515, 2002.

Peter C. Fishburn. Condorcet social choice functions. SIAM Journal on Applied Mathematics, 33(3):469-489, 1977.

Peter C. Fishburn. Monotonicity paradoxes in the theory of elections. Discrete Applied Mathematics, 4(2):119-134, 1982.

Peter C. Fishburn and Steven J. Brams. Paradoxes of preferential voting. Mathematics Magazine, 56(4):207-214, 1983.

Peter C. Fishburn and Steven J. Brams. Manipulability of voting by sincere truncation of preferences. Public Choice, 44(3):397-410, 1984.

Nicolas Houy. A characterization of majority voting rules with quorums. Theory and Decision, 67(3):295-301, 2009.

Jose L. Jimeno, Joaquín Pérez, and Estefanía García. An extension of the Moulin no show paradox for voting correspondences. Social Choice and Welfare, 33(3): 343-359, 2009.

Hervé Moulin. The Strategy of Social Choice. North-Holland, Amsterdam, New York, 1983.

Hervé Moulin. Condorcet's principle implies the no show paradox. Journal of Economic Theory, 45(1):53-64, 1988.

Hervé Moulin. Axioms of Cooperative Decision Making. Cambridge University Press, 1991.

Roger Myerson. Axiomatic derivation of scoring rules without the ordering assumption. Social Choice and Welfare, 12:59-74, 1995.

Hannu Nurmi. Voting Paradoxes and How to Deal with Them. Springer, 1999.

Joaquín Pérez. The strong no show paradoxes are a common flaw in Condorcet voting correspondences. Social Choice and Welfare, 18:601-616, 2001.

Jeffrey T. Richelson. Running off empty: run-off point systems. Public Choice, 35 (4):457-468, 1980.

Donald G. Saari. Consistency of decision processes. Annals of Operations Research, 23(1):103-137, 1990. 
M. Remzi Sanver and William S. Zwicker. One-way monotonicity as a form of strategy-proofness. International Journal of Game Theory, 38(4):553-574, 2009.

John Smith. Aggregation of preferences with a variable electorate. Econometrica, 41:1027-1041, 1973.

H. Peyton Young. An axiomatization of Borda's rule. Journal of Economic Theory, 9(1):43-52, 1974.

H. Peyton Young. Social choice scoring functions. SIAM Journal of Applied Mathematics, 27:824-838, 1975. 